\title{
High resolution neutron spectrometry with liquid scintillation detectors for fusion applications
}

Andreas Zimbal ${ }^{*}$, Horst Klein, Marcel Reginatto, Helmut Schuhmacher

Physikalisch-Technische Bundesanstalt

Bundesallee 100, D-38116 Braunschweig, Germany

E-mail: Andreas .Zimbal@PTB.DE

\section{Luciano Bertalot}

Associazione Euratom-ENEA sulla Fusione

C.P. 65, I-00044 Frascati, Roma, Italy

\section{Andrea Murari}

Consorzio RFX-Ass. Euratom-ENEA sulla Fusione

Corso Stati Uniti 4, I-35127 Padua, Italy

and JET-EFDA Contributors

See annex of J. Pamela et al., 2003 Overview of recent JET results, Nucl. Fusion 43

International Workshop on Fast Neutron Detectors

University of Cape Town, South Africa

April 3-6, 2006

\footnotetext{
${ }^{*}$ Speaker
} 
Organic liquid scintillation detectors, e.g. NE213, EJ-301 or BC501A, are routinely used as high resolution neutron spectrometers in many nuclear experiments. The neutron energy spectrum can be reliably determined from the pulse height spectrum provided the response function of the detector is well determined, counting statistics is sufficient, a suitable unfolding method is applied and care is taken to ensure the gain stability of the system during the measurement. The Physikalisch-Technische Bundesanstalt (PTB) has many years of experience with the development, detailed characterization and use of liquid scintillation detectors.

Recent work done at PTB in collaboration with ENEA and EFDA-JET concerning the use of liquid scintillation detectors as neutron diagnostic instruments in fusion experiments is discussed. An NE213 liquid scintillator system has been carefully characterized and successfully used in a series of measurements at the Joint European Torus (JET). We describe the improvements that were made to the system to meet the challenging measurement conditions at JET, as well as the processes used to calibrate and characterize the system. For the determination of the neutron light output function and for a comparison of experimentally determined response functions with those calculated with Monte Carlo techniques, measurements have been performed at the PTB facility in mono-energetic neutron reference fields and in fields with a broad energy distribution applying time of flight spectrometry. Selected examples of measured neutron spectra are discussed in terms of energy resolution and compared to the expected neutron spectra, both for measurements at the PTB accelerator and at JET. 


\section{Introduction}

Organic liquid scintillation detectors such as NE213, EJ-301 or BC501A are routinely used as spectrometers for neutron and photon radiation in many nuclear experiments. The intrinsic properties of these scintillators permit the separation of neutron and photon induced events by pulse shape analysis and allow for spectrometry in mixed neutron/photon radiation fields [1].

The possibility of using these detectors as high resolution neutron spectrometers, e.g. at fusion experiments like the Joint European Torus (JET), has been demonstrated in recent experimental campaigns [2]. For these applications it is necessary to ensure gain stability of the system and to know precisely the detector response function needed for the application of dedicated methods of data analysis [3]. The envisaged installation of compact neutron detection systems at the International Thermonuclear Experimental Reactor (ITER) require further developments in the field of data acquisition $[4,5]$ as well as the investigation of alternative methods for the characterization of the detectors.

\section{The NE213 scintillation detector}

For the experiments at JET [2], an NE213 scintillation detector $(5.08 \mathrm{~cm}$ in diameter and in length) was used. The scintillator cell is coupled through a partially coated light guide to an XP2020 Photomultiplier. In this way, the light collection system is designed for an optimized pulse height resolution [6]. An LED signal fed into the light guide was used as a monitor for count rate dependent gain variations of the photomultiplier. Neutron spectrometry in the energy range from $1.5 \mathrm{MeV}<E_{\mathrm{n}}<20 \mathrm{MeV}$ and photon spectrometry in the energy range from $0.2 \mathrm{MeV}<E_{\gamma}<10 \mathrm{MeV}$ can be performed with this detector system.

\section{Determination of the detector response functions for neutrons}

The approach used up to now at PTB for the characterization of scintillation detectors $[1,7]$ is based on a combination of measurements in mono-energetic neutron and photon reference fields and Monte Carlo calculations using specially developed programs [8]. The calculations are partly based on parameters which need to be determined from the experimental results. Although this method has proven to be very powerful, there are certain limitations to it. Small deficiencies in the response functions used for the unfolding can result in oscillations in the resulting neutron spectra in the case of broad neutron energy distributions or in spurious peaks in the case of nearly mono-energetic neutron spectra. We report on another calibration technique $[9,10]$ that improves upon the standard approach.

A neutron beam with a broad energy distribution was produced using the ${ }^{9} \mathrm{Be}(\mathrm{p}, \mathrm{n})$-reaction with the ns-pulsed proton beam $\left(E_{\mathrm{p}}=19 \mathrm{MeV}\right)$ from the PTB cyclotron and a thick $(3 \mathrm{~mm})$ Be-target. The spectral neutron distribution of this reaction determined with the time-of-flight technique (TOF) is shown in Figure 1. 


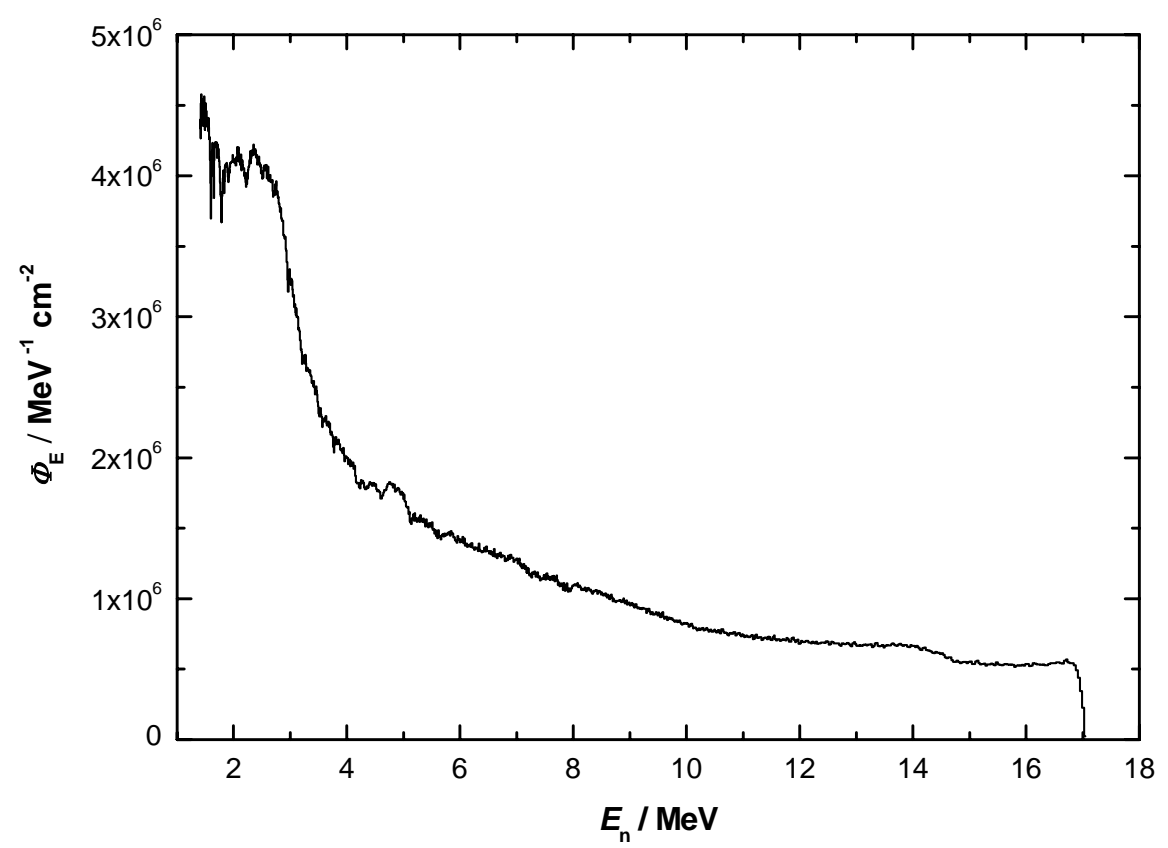

Figure 1: Spectral fluence of neutrons $\Phi_{\mathrm{E}}$ from of the ${ }^{9} \mathrm{Be}(\mathrm{p}, \mathrm{n})$-reaction using the ns-pulsed proton beam $\left(E_{\mathrm{p}}=19 \mathrm{MeV}\right)$ from the PTB cyclotron and a thick $(3 \mathrm{~mm})$ Be-target analyzed from a time-of-flight measurement.

The NE213 detector was placed at a distance of $27.35 \mathrm{~m}$ from the target behind a collimator. During a $40 \mathrm{~h}$ run, the amplitude of the integrated signal from the $9^{\text {th }}$ dynode of the photomultiplier tube (PMT), the shape of the anode pulse and the TOF of each event were analyzed with conventional NIM standard electronic modules and stored in list mode using a MPA-3* multi parameter data acquisition system. The TOF information was recorded with 8192 channels using a time calibration of $0.5 \mathrm{~ns} /$ channel, the pulse height converted with a gain factor of $5 \mathrm{keV}_{\mathrm{ee}} /$ channel obtained by a calibration with a ${ }^{207} \mathrm{Bi} \gamma$-source in terms of electron equivalent energies and the pulse shape information were recorded with 4096 channels each. From these data, a two-dimensional histogram was extracted representing the pulse height response of the detector as a function of the time-of-flight. This two-dimensional histogram is shown in Figure 2, where all neutron and photon events are reported. The uncertainty in the determination of the neutron energy can be estimated to be $58 \mathrm{keV}$ for a neutron energy of $14 \mathrm{MeV}$ taking into account the width of the prompt $\gamma$-peak in the TOF spectrum, the time calibration of the Time to Amplitude Converter used in this experiment (including differential nonlinearity and calibration against a DCF77 based frequency standard) and the time response of the detector chiefly determined by its length.

A total of 1061 time slices corresponding to neutron energies between $1.5 \mathrm{MeV}$ and 17.0 MeV were extracted from the pulse height response of the detector shown in Figure 2, after gating to neutron induced events only and after correction of the "satellite" peaks from the

\footnotetext{
${ }^{*}$ www.fastcomtec.com
} 
cyclotron. The energy width of the channels in the neutron time of flight spectrum increases with neutron energy (from $8 \mathrm{keV}$ at $E_{\mathrm{n}}=2.5 \mathrm{MeV}$ to $27 \mathrm{keV}$ at $E_{\mathrm{n}}=14 \mathrm{MeV}$ ) and is a factor of 15 smaller than the resolution of the corresponding pulse height spectrum even for the highest neutron energies, which is important for achieving an energy resolution 4-5 times smaller than the corresponding pulse height resolution with unfolding methods. After fluence normalization, these 1061 individual functions for mono-energetic neutrons form the response matrix needed for the unfolding process. The NRESP7 code [8] was used for the fluence normalization of these pulse height spectra, considering only the pulse height range resulting from $(n, p)$-scattering (see Figure 3). The threshold for this normalization was varied with the neutron energy. The proton light output function of the detector, an important input for the NRESP7 calculations, was also extracted from the 1061 pulse height spectra. The result of this analysis is shown in Figure 3. The measured response matrix was then used for the unfolding of data collected in the PTB reference neutron fields (section 4) and at JET (section 5).

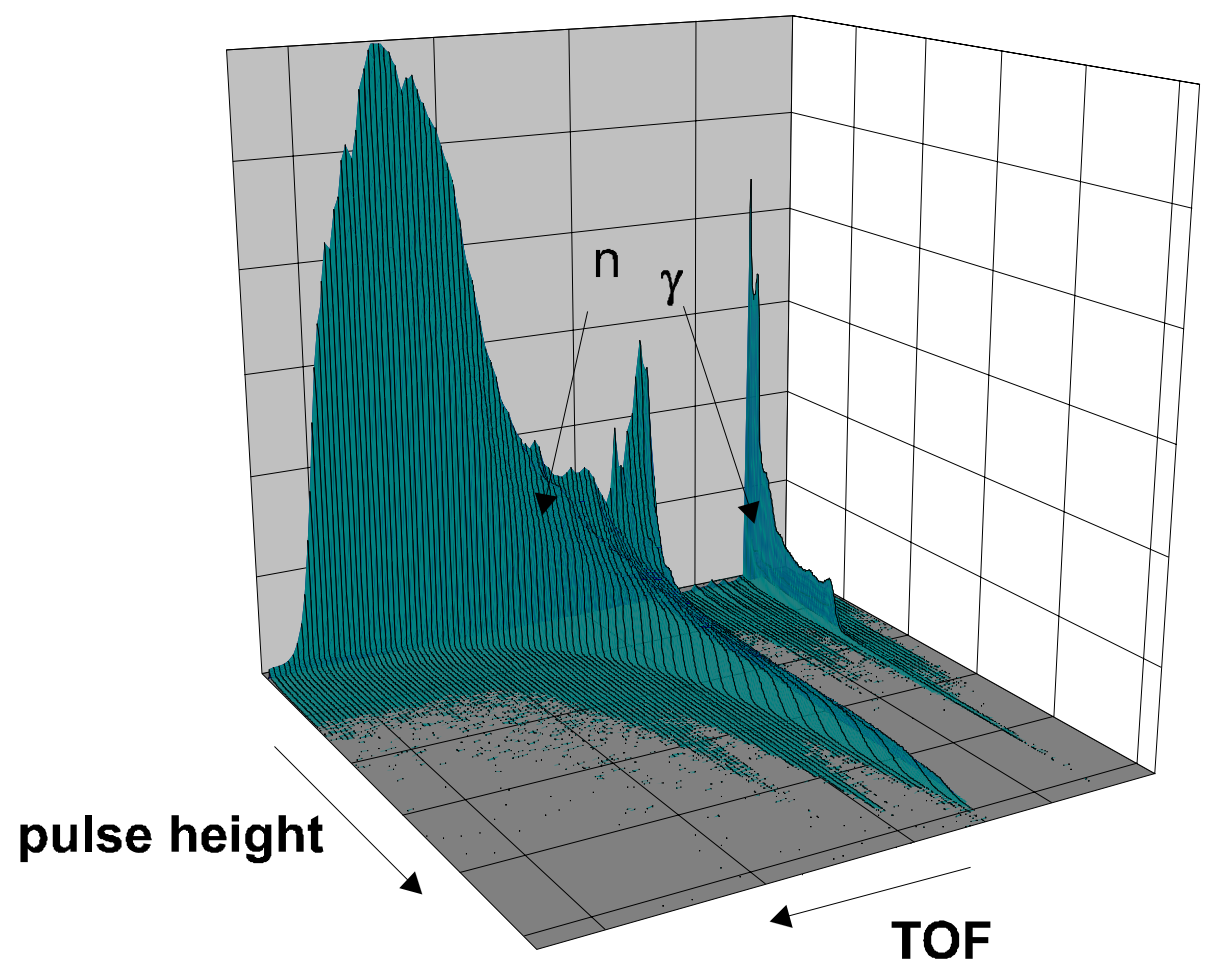

Figure 2: Pulse height response of the detector vs. TOF measured with the ${ }^{9} \mathrm{Be}(\mathrm{p}, \mathrm{n})$-reaction. The prompt $\gamma$-peak and the neutron TOF-distribution can clearly be seen, but also contributions from "satellite"-pulses of the cyclotron which can be identified in the TOF-region between the prompt $\gamma$-peak and the neutron events, or at high pulse amplitudes for long TOF, respectively. The detector response matrix was extracted from this data set selecting only neutron events by pulse shape analysis. In total $9.0 \cdot 10^{7}$ neutron events were recorded during a $40 \mathrm{~h}$ run. 

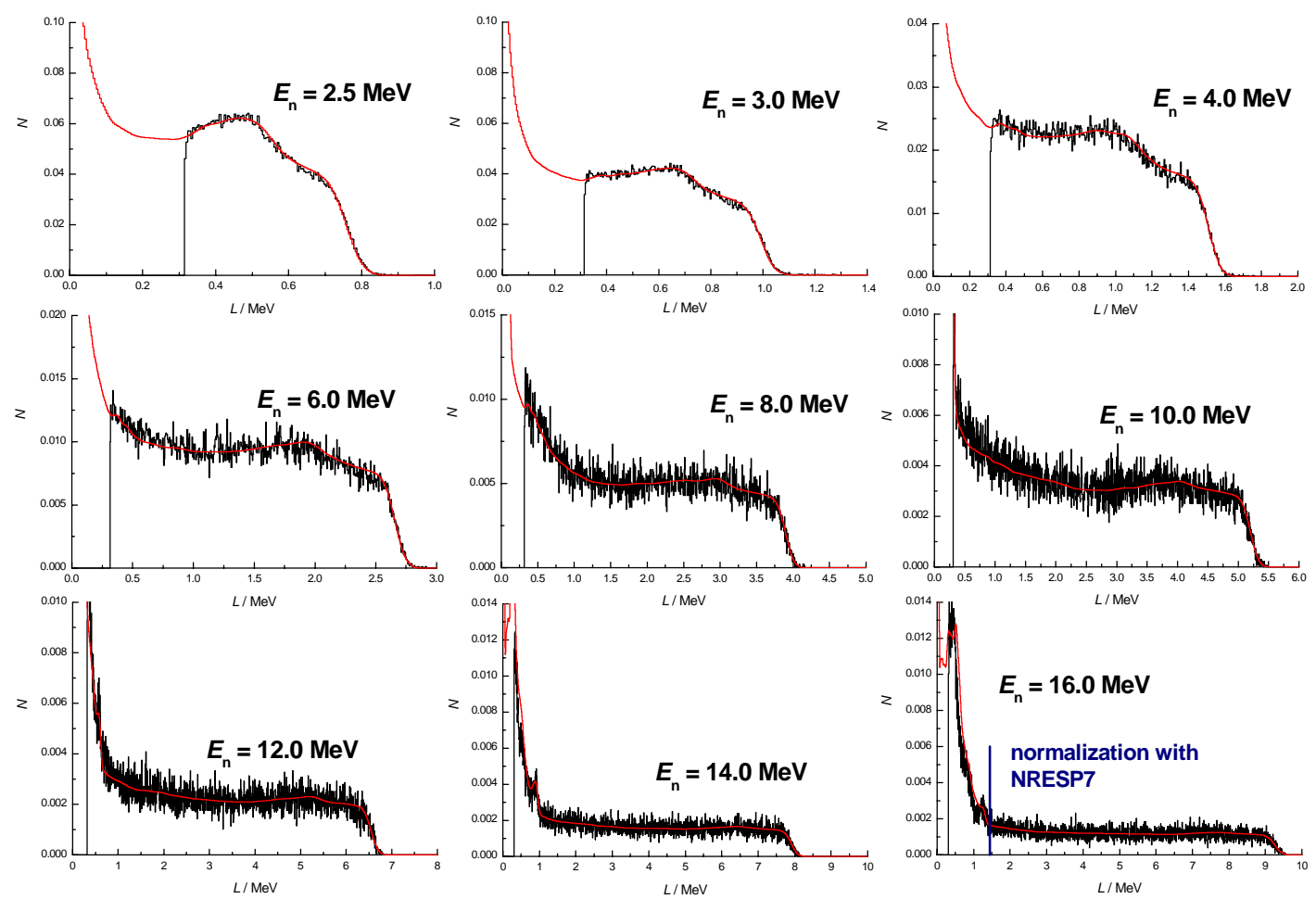

Figure 3: Response functions of the NE213 scintillation detector for 9 neutron energies selected between $2.5 \mathrm{MeV}$ and $16 \mathrm{MeV}$ by time-of-flight slices. The experimental spectra (black histogram) are compared with and normalized to responses calculated with the NRESP7 code (red lines).

\section{Test in PTB reference fields}

Measurements in mono-energetic neutron reference fields were carried out at the PTB accelerator facility to test the new calibration procedure of the detector. Among the different neutron fields available at PTB, two specific reactions provide the opportunity of producing mono-energetic neutron beams with energies of $2.5 \mathrm{MeV}$ and $14 \mathrm{MeV}$, i.e. nearly the same energy as the neutrons of nuclear fusion discharges and in particular with line widths comparable to line widths expected for a thermal fusion plasma with a Maxwellian temperature around $1 \mathrm{keV}$. Due to these conditions, the measurements performed at PTB are an excellent test of the diagnostics capabilities of this neutron spectrometer for fusion application.

The energy of neutrons produced in the reactions $\mathrm{D}(\mathrm{d}, \mathrm{n})$ and $\mathrm{T}(\mathrm{d}, \mathrm{n})$ in the laboratory frame is shown in Figure 4 for different incoming deuteron energies. The targets used at PTB to produce neutrons from these reactions are $1-2 \mathrm{mg} / \mathrm{cm}^{2}$ thick deuterium and tritium loaded Ti-targets on a Ag backing in which the deuterons are fully stopped. The neutrons produced are therefore not mono-energetic except for one emission angle in the laboratory frame, which can be identified in Figure 4 as the crossing point of the kinematics for different deuteron beam energies, i.e. $106^{\circ}$ in case of the $\mathrm{D}(\mathrm{d}, \mathrm{n})$-reaction and $98^{\circ}$ in case of the $\mathrm{T}(\mathrm{d}, \mathrm{n})$-reaction. The very 

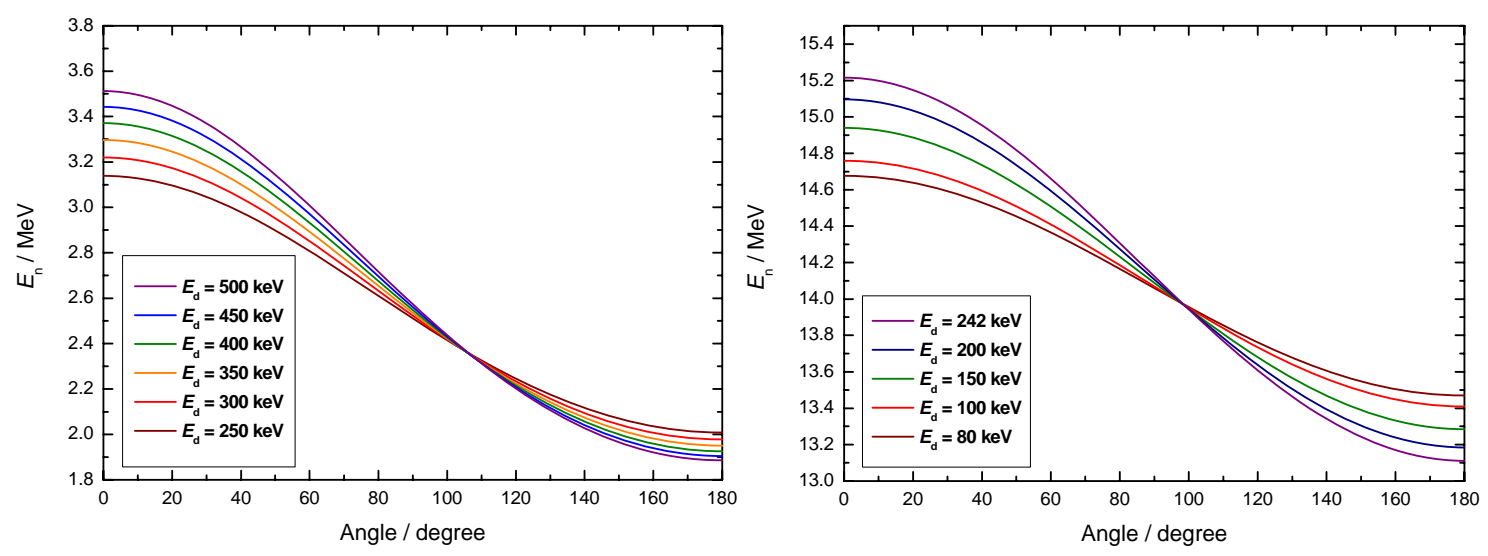

Figure 4: Kinematics of the $\mathrm{d}+\mathrm{D} \rightarrow{ }^{3} \mathrm{He}+\mathrm{n} \quad$ (left) and the $\mathrm{d}+\mathrm{T} \rightarrow{ }^{4} \mathrm{He}+\mathrm{n} \quad$ (right) reaction in the laboratory frame. The neutron energies considered here are those relevant for the solid Ti-targets used at PTB with an energy of the incoming deuteron beam of $E_{\mathrm{d}}=500 \mathrm{keV}$ for the $\mathrm{D}(\mathrm{d}, \mathrm{n})$ and of $E_{\mathrm{d}}=242 \mathrm{keV}$ for the $\mathrm{T}(\mathrm{d}, \mathrm{n})$ case.

small energy distribution resulting from the kinematics of the reaction is broadened due to angle scattering of the incoming deuterons in the Ti-target. Calculations of the neutron energy distribution for the targets used have been performed with the TARGET ${ }^{\dagger}$ code [11] and the results are shown in Figures 5 and 6 (thin lines) for the $D(d, n)$ and the $T(d, n)$ case.

Pulse height spectra (PHS) were been acquired in these neutron reference fields. To ensure the stability of the detector system in terms of energy calibration and pulse height resolution, measurements with a ${ }^{207} \mathrm{Bi} \gamma$-source were made regularly. The measured $\gamma$-PHS were analyzed with the same procedures that were used during the determination of the response matrix. The neutron PHS were unfolded with the maximum entropy deconvolution code MAXED [12, 13] using the measured response matrix (section 3) and "flat" default spectra (i.e. default spectra which did not contain any information of the peak structure expected for the solution spectra). The $\chi^{2}$-value of the solution was determined with a method ("L-curve method") described briefly in [2]. The results of the unfolding for the different angles are shown by the thick lines in Figures 5 and 6.

In the $\mathrm{D}(\mathrm{d}, \mathrm{n})$ case (Figure 5), the TARGET calculation for $106^{\circ}$ results in a Gaussian shaped distribution with a full width at half maximum (FWHM) of $84 \mathrm{keV}$, whereas the unfolded measurement results in a value of $79 \mathrm{keV}$. In the case of the measurement at $0^{\circ}$, the shape of the calculation is confirmed in the unfolding, except for a small shift of the high energy edge of the distribution. The reason for this in not clear up to now and might be due to some unknown properties of the neutron producing target, namely the density distribution of the deuterium-atoms in the Ti-layer, which will influence the energy distribution of the neutron beam much more in the $0^{\circ}$ case than under $106^{\circ}$.

\footnotetext{
${ }^{\dagger}$ The authors would like to thank Dr. D. Schlegel for performing the calculations of the expected line shapes for the different experimental settings with the TARGET calculation tool.
} 


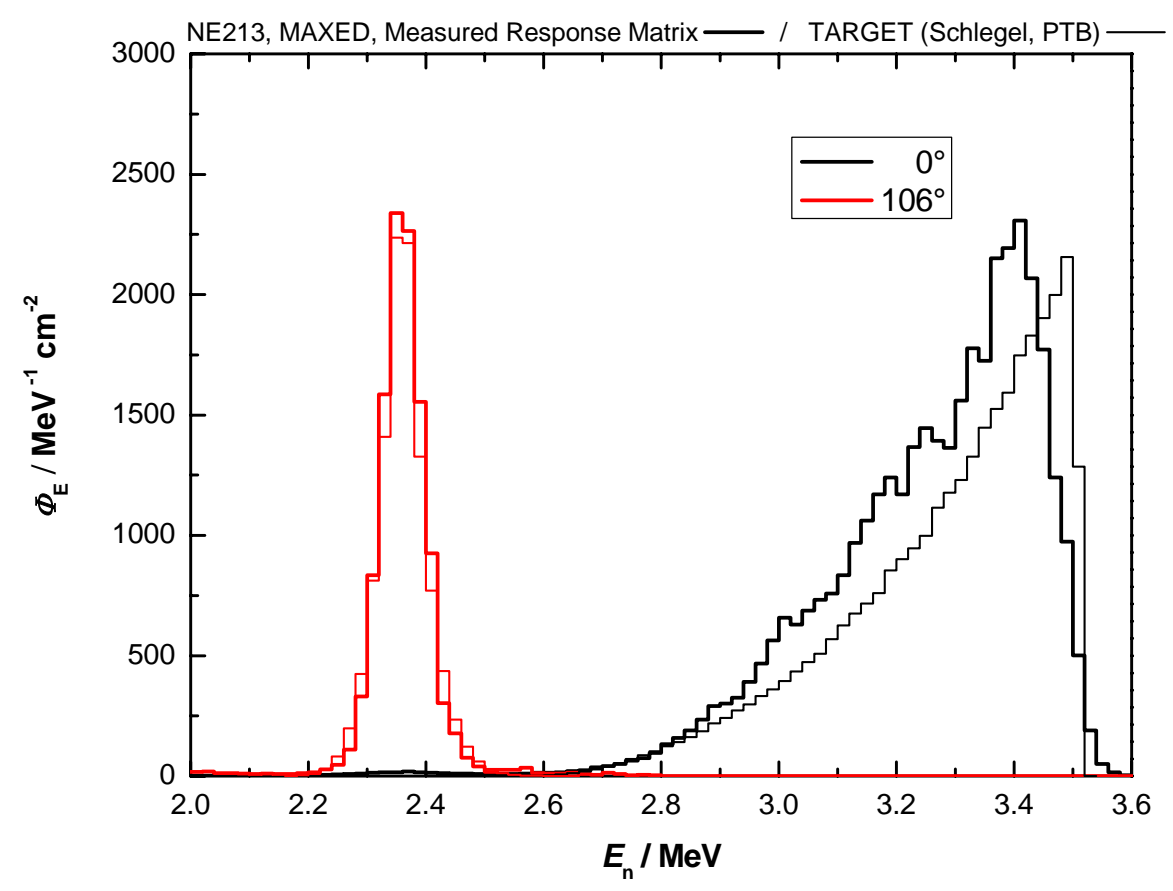

Figure 5: Calculated (thin line) and measured (thick line) energy distribution for neutrons using the $\mathrm{d}+\mathrm{D} \rightarrow{ }^{3} \mathrm{He}+\mathrm{n}$ reaction at different positions of the detector (neutron emission angles of $106^{\circ}$ and $0^{\circ}$ relative to the incoming deuteron beam). The unfolding was performed using the experimentally determined response matrix (see section 3 ).

The same comparison was performed for the $T(\mathrm{~d}, \mathrm{n})$ case (Figure 6). In this case, the TARGET calculation gives a value of $200 \mathrm{keV}$ for the $F W H M$ at $98^{\circ}$, whereas the unfolding gives a significant larger value of $425 \mathrm{keV}$. The measurements and calculations for $0^{\circ}$ agree very well. The reason for the discrepancy between TARGET calculation and measurement under $98^{\circ}$ is believed to be the result of the low statistics of the measured response functions, which is not as good for neutrons with energies around $14 \mathrm{MeV}$ as for neutrons with energies around $2.5 \mathrm{MeV}$ (see Figure 3). This view is supported by the fact that much better agreement between the TARGET calculation and measurement under $98^{\circ}$ is obtained if a response function purely based on the NRESP7 Monte Carlo calculations (red lines in Figure 3) is used for the unfolding. The result of this unfolding is shown in Figure 7. In this case, the FWHM of the TARGET calculation and the unfolded measurement for the $98^{\circ}$ case agree very well $(200 \mathrm{keV}$ respectively $220 \mathrm{keV}$ ). The calculated response matrix has the advantage of resolving smaller structures in the results of the unfolding. But, as can be seen in Figure 7, spurious peaks appear at the low energy side of the $14 \mathrm{MeV}$ neutron peak, which are due to the imperfect calculated response matrix [1]. The problem with the low statistics in the measured response functions can be solved by either making measurements for the determination of the detector response functions with longer counting times or by using methods which remove the noise due to the statistics, e.g. applying smoothing procedures or introducing smooth parameterized functions that can be fitted to the high energy part of the response. 


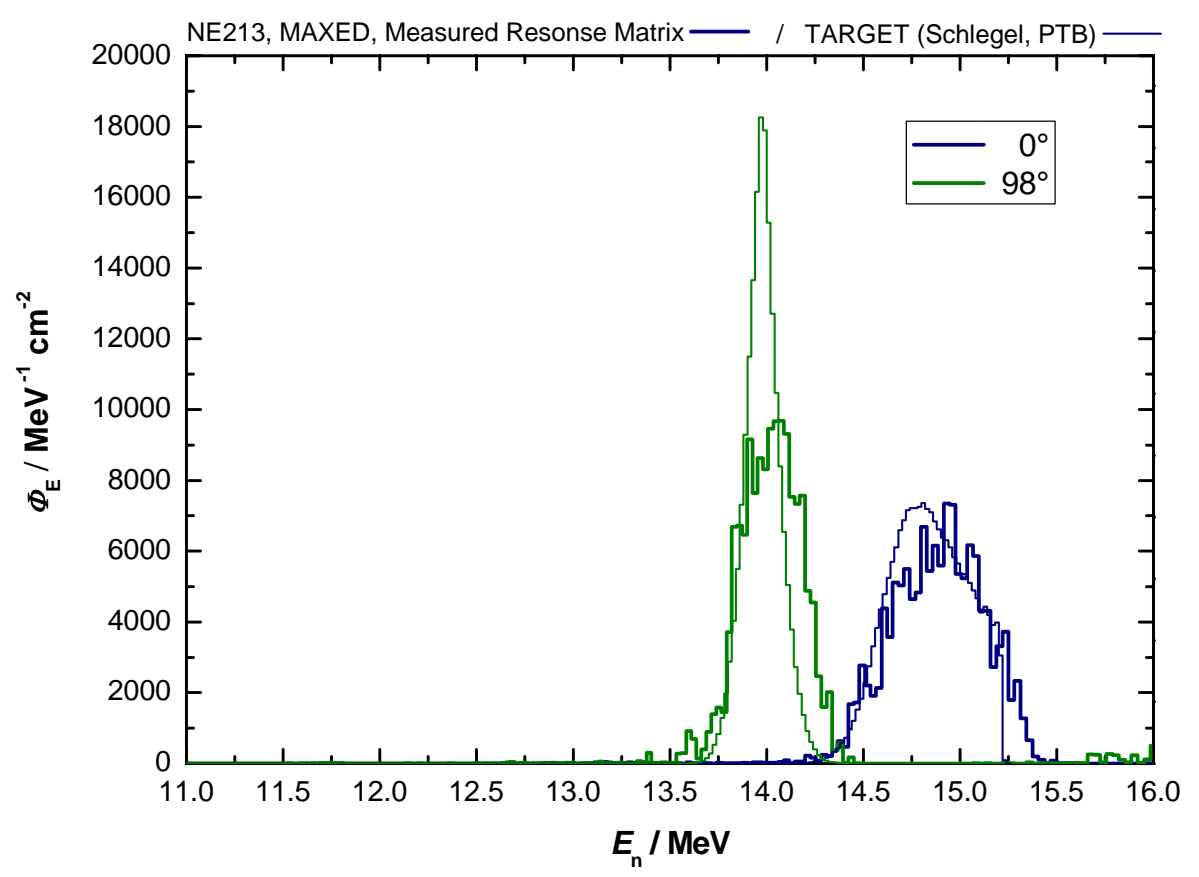

Figure 6: Calculated (thin lines) and measured (thick line) energy distribution for neutrons using the $\mathrm{d}+\mathrm{T} \rightarrow{ }^{4} \mathrm{He}+\mathrm{n} \quad$ reaction at different positions of the detector (neutron emission angles of $98^{\circ}$ and $0^{\circ}$ relative to the incoming deuteron beam). The unfolding was performed using the experimentally determined response matrix (see section 3).

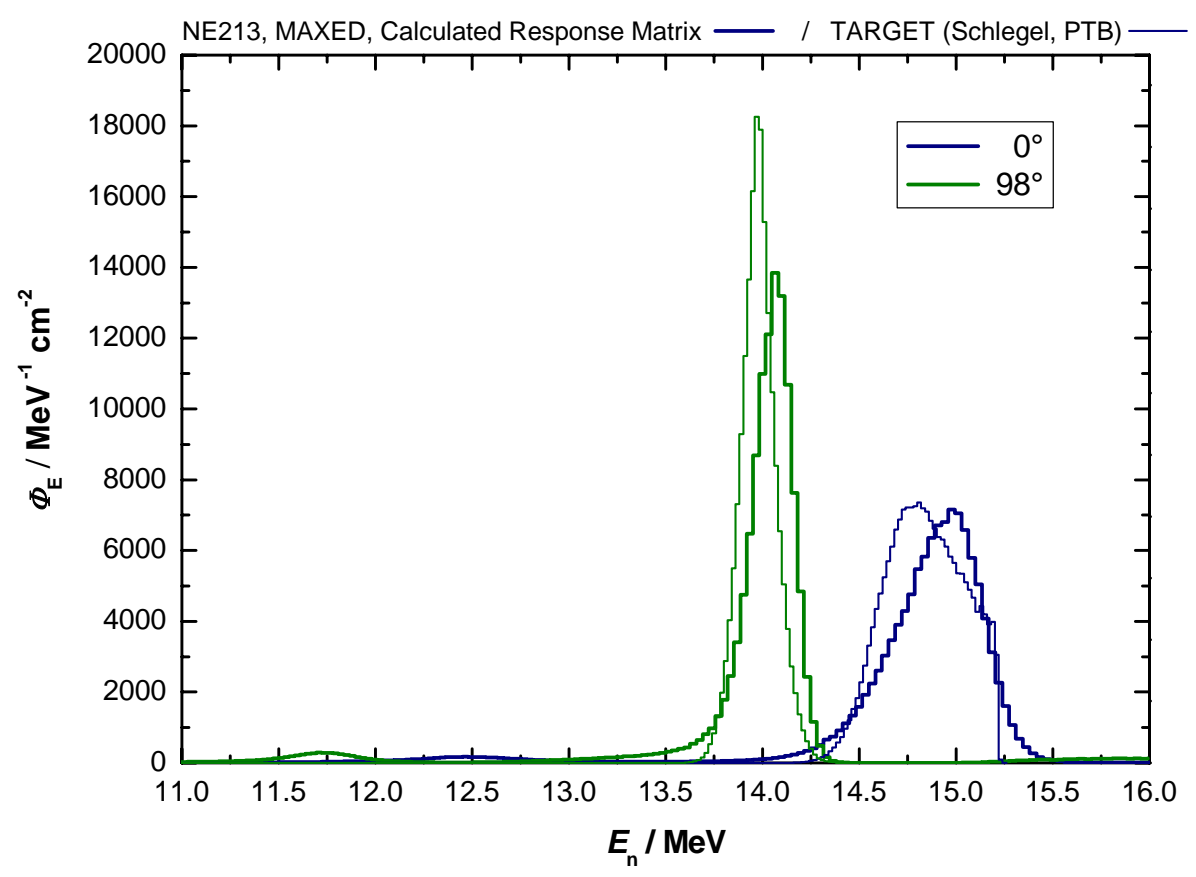

Figure 7: Calculated (thin lines) and measured (thick lines) energy distribution for neutrons using the $\mathrm{d}+\mathrm{T} \rightarrow{ }^{4} \mathrm{He}+\mathrm{n}$ reaction at different positions of the detector. The unfolding was performed using the calculated response matrix (red lines in Figure 3). 


\section{Measurements at the Joint European Torus (JET)}

During two campaigns in 2002 and 2003 at the Joint European Torus (JET), the NE213 detector was installed in the "roof lab" with a vertical line of sight, at a distance of $19 \mathrm{~m}$ from the centre of the plasma. Pulse height spectra (PHS) were measured for various heated plasma scenarios [2]. The analysis of these data with the newly determined response matrix illustrates the capabilities of this spectrometer for high resolution neutron spectrometry in fusion experiments. The PHS have been unfolded with the MAXED code using "flat" default spectra (i.e. with default spectra which do not contain any information of the peak structure expected for the solution). Examples of such data analysis are shown in Figures 8 and 9.

For pure ohmic heating of the plasma, the expected shape of the neutron energy distribution corresponds to a Gaussian distribution with a FWHM related to the ion temperature $\left(T_{\mathrm{i}}\right)$ of the plasma as follows: $F W H M=88.2 \cdot \sqrt{T_{\mathrm{i}}} \mathrm{keV}^{1 / 2}\left(T_{\mathrm{i}}\right.$ in $\left.\mathrm{keV}\right)$ [14]. The result of the unfolding of the measured PHS (shown as black histogram in the insert of Figure 8) is a Gaussian shaped line with a FWHM of $126 \mathrm{keV}$. The ion temperature calculated from this value results in $T_{\mathrm{i}}=2.3 \mathrm{keV}$. From other plasma diagnostics ("charge exchange" measurements) it is known that a typical value for this kind of plasma scenario is $T_{\mathrm{i}}=2.0 \mathrm{keV}$, which leads to good agreement between these two independent methods.

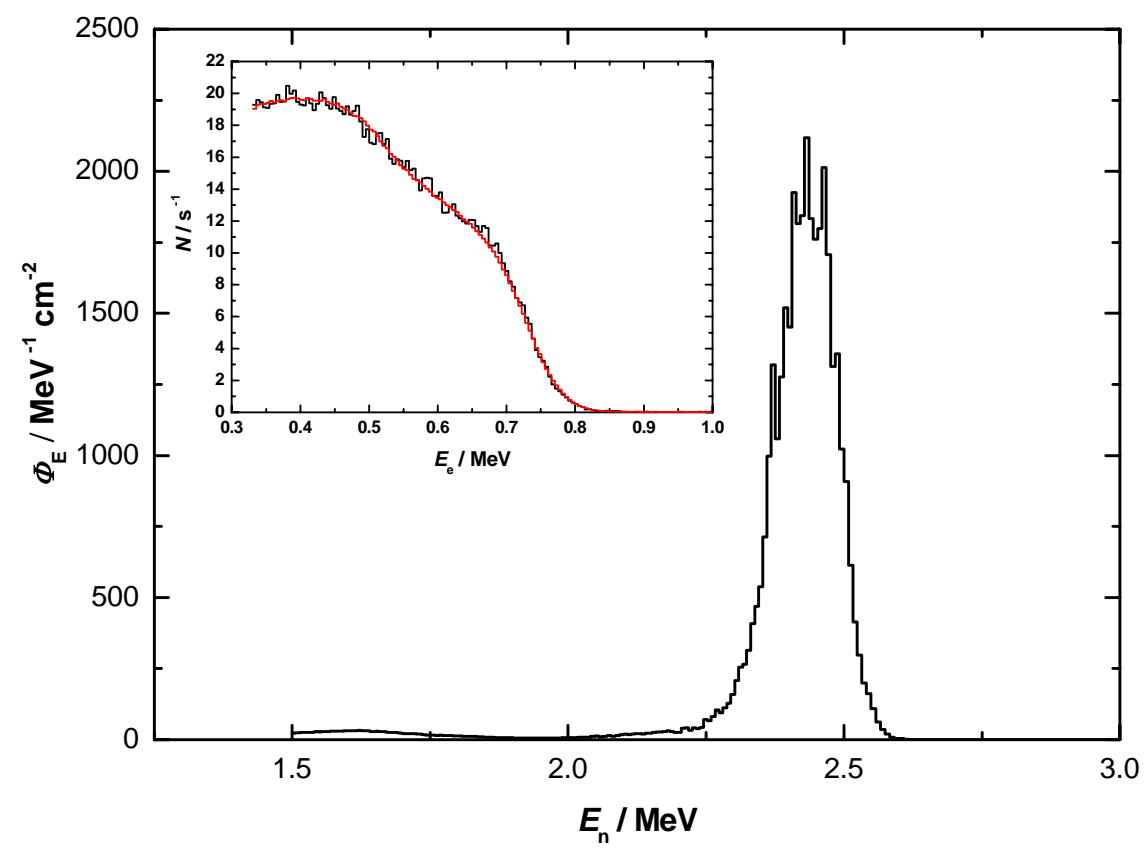

Figure 8: Pure ohmic heating. The insert shows the measured PHS (black) as well as the PHS that results from folding the analyzed neutron spectrum with the response functions (red) [JET pulse No. $57112 \ldots 57117+57151 \ldots 57153(52.7$ s - 59.7 s), 56 s total @ 1700 counts per second (cps)]. 
For other heating scenarios, like neutral beam injection (NBI) or radio frequency (RF) heated plasmas, the expected line shape is not Gaussian but shows different structures in the neutron energy distribution which are produced by the non thermal, i.e. high energy, population of ions in the plasma. As an example, the measurement of an ohmic and NBI heated plasma is shown in Figure 9. The shape of the fusion peak shows a broad distribution with a FWHM of around $575 \mathrm{keV}$, which is in agreement with the result of calculations $(530 \mathrm{keV}$ [15]).

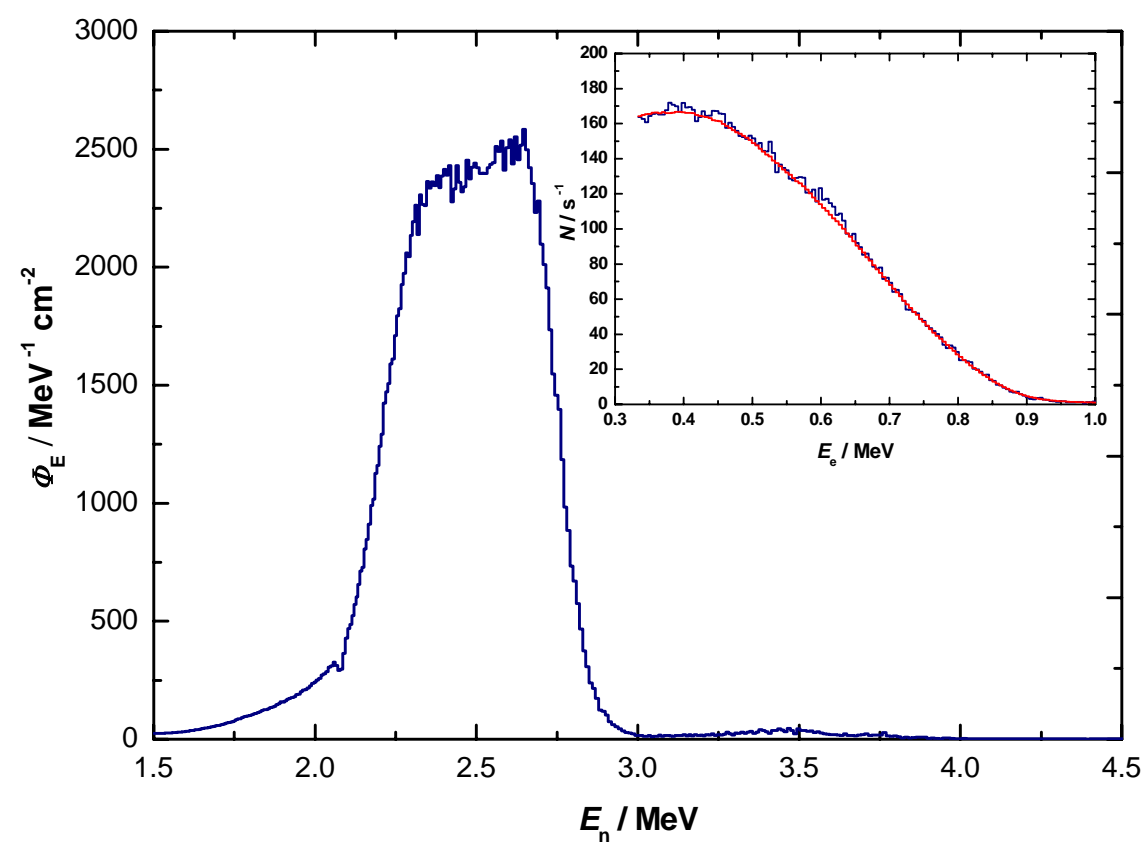

Figure 9: Ohmic + NBI heating. The insert shows the measured PHS (blue) as well as the PHS that results from folding the analyzed neutron spectrum with the response functions (red) [JET pulse No. 61039 (53.0 s-64.0 s), 11 s total @ 16000 cps].

\section{Conclusions}

The application at JET of liquid scintillation detectors, well characterized at the PTB facilities, has shown that these detectors can be used as high resolution compact neutron spectrometers in fusion research. The new calibration technique using the broad energy distribution of the ${ }^{9} \mathrm{Be}(\mathrm{p}, \mathrm{n})$-reaction allows one to determine experimentally the detector response function which plays a key role in the unfolding methods used for the determination of the neutron energy spectra. This is a clear advantage as compared to the methods used at PTB up to now. Future activity will be performed at JET and PTB on the use of liquid scintillators with fast digital acquisition to optimize the system for higher count rates and further investigations will be done to study the influence of the statistics in both the PHS and in the response matrix on the results of the unfolding. 


\section{References}

[1] H. Klein, S. Neumann, Neutron and photon spectrometry with liquid scintillation detectors in mixed fields, Nuclear Instruments and Methods in Physics Research A 476 (2002) 132-142

[2] A. Zimbal et. al., Compact NE213 neutron spectrometer with high energy resolution for fusion applications, Review of Scientific Instruments, Vol. 75 No. 10 (2004) 3553

[3] M. Reginatto, A. Zimbal, Bayesian methods for the analysis of pulse height spectra from liquid scintillation detectors, to be published

[4] B. Esposito et. al., Neutron measurements on Joint European Torus using an NE213 scintillator with digital pulse shape discrimination, Review of Scientific Instruments, Vol. 75 No. 10 (2004) 3550

[5] D. Marocco et. al., A digital data acquisition system optimized for spectrometry with liquid scintillation detectors, these proceedings

[6] H. Klein, H. Schölermann, Improvement of the light collection in scintillation detectors, IEEE transactions on Nuclear Science, Vol. NS-26, No. 1 (1979) 373

[7] D. Schmidt, B. Asselineau, R. Böttger, H. Klein, L. Lebreton, S. Neumann, R. Nolte, G. Pichenot, Characterization of liquid scintillation detectors, Nuclear Instruments and Methods in Physics Research A 476 (2002) 186-189

[8] G. Dietze and H. Klein, NRESP4 and NEFF4: Monte Carlo Codes for the Calculation of Neutron Response Functions and Detection Efficiencies for NE213 Scintillation Detectors, PTB Report PTB-ND-22, 1982, ISSN 0572-7170

[9] E. Dekempeneer et al., A spectrometer for double-differential neutron-emission cross section measurements in the energy range 1.6 to $16 \mathrm{MeV}$, Nuclear Instruments and Methods in Physics Research A 256 (1987) 489-498

[10] A. Hildebrand, H. Park, S. Khurana, R. Nolte, D. Schmidt, Experimental Determination of the Response Matrix of a BC501A Scintillation Detector using a Wide Neutron Spectrum: A Status Report, Laboratory Report PTB-6.42-05-1, 2005

[11]D. Schlegel, Target User's Manual, Laboratory Report PTB-6.42-05-2, 2005

[12] M. Reginatto, P. Goldhagen, MAXED, A Computer Code for Maximum Entropy Deconvolution of Multisphere Neutron Spectrometer Data, Health Phys. 77 (1999) 579-583

[13] M. Reginatto, P. Goldhagen, S. Neumann, Spectrum unfolding, sensitivity analysis and propagation of uncertainties with the maximum entropy deconvolution code MAXED, Nuclear Instruments and Methods in Physics Research A 476 (2002) 242-246

[14] L. Ballabio, G. Gorini, J. Kallne, Energy spectrum of thermonuclear neutrons, Review of Scientific Instruments, Vol. 68 No. 1 (1997) 585

[15] L. Bertalot et. al., Neutron energy measurements of trace tritium plasmas with NE213 compact spectrometer at JET, Europhysics Conference Abstracts Vol. 29C (2005) http://eps2005.ciemat.es/papers/pdf/P1_078.pdf 